\title{
Immunmodulation bei autoimmunen interstitiellen Lungenerkrankungen
}

\author{
Benjamin Seeliger ${ }^{a}$ Antje Prasse ${ }^{a, b}$ \\ ${ }^{a}$ Abteilung für Pneumologie, Medizinische Hochschule Hannover und Biomedical Research in End-stage and Obstructive Lung Disease \\ Hannover (BREATH), Deutsches Zentrum für Lungenforschung (DZL), Hannover, Deutschland; ${ }^{b}$ Fraunhofer-Institut für Toxikologie und \\ Experimentelle Medizin, Hannover, Deutschland
}

\section{Schlüsselwörter}

Immunmodulation · interstitielle Lungenerkrankung .

Immunsuppression

\section{Zusammenfassung}

Interstitielle Lungenerkrankungen (ILD) im Rahmen einer Autoimmun- oder Systemerkrankung werden zunehmend erkannt, und der Kenntnisstand zu ihrer Pathophysiologie erweitert sich derzeit rapide. Doch das therapeutische Vorgehen stützt sich weiterhin vor allem auf etablierte langwirksame Antirheumatika (DMARD), zu denen - trotz ihrer jahrzehntelangen Anwendungsgeschichte bei zugrunde liegenden Erkrankungen wie rheumatoider Arthritis vor allem retrospektive Daten vorliegen, um Aufschluss über ihre Auswirkungen auf den Verlauf der ILD zu geben. Randomisierte
Studien zur Untersuchung der Auswirkungen von biologischen DMARD sind in den letzten Jahren abgeschlossen worden oder laufen derzeit und bringen so neue Behandlungsoptionen für diese oft erbarmungslos fortschreitenden Erkrankungen hervor. Hier geben wir einen Überblick über die Evidenzlage und die gegenwärtige Anwendungspraxis von synthetischen und biologischen DMARD bei ILD im Zusammenhang mit Autoimmun- und systemischen Erkrankungen. (c) 2021 S. Karger AG, Basel

\section{Einleitung}

Die interstitiellen Lungenerkrankungen (ILD) umfassen ein Spektrum von Erkrankungen, die entweder durch entzündliche oder fibrosierende Prozesse oder beides gekennzeichnet sind. Patienten mit idiopathischer pulmonaler Fibrose (IPF) [1] haben in der Landmark-Studie PANTHER [2] unerwünschte Reaktionen auf Immunsuppression gezeigt; somit ist die Immunsuppression bei IPF in Abwesenheit akuter Exazerbationen oder inflammatorischer Komorbidität kontraindiziert [3]. Ein großer Teil der ILD tritt jedoch im Rahmen bestehender Autoimmun- oder Bindegewebserkrankungen (CTD; connective tissue diseases) auf, wie z.B. systemische Sklerose (SSc), rheumatoide Arthritis (RA) oder idiopathische inflammatorische Myositis (IIM); hier richtet sich die Therapie primär nach den Anzeichen der jeweils zugrunde liegenden Erkrankung. Darüber hinaus zeigen einige Patienten mit fibrotischen Lungenerkrankungen Anzeichen von Autoimmuni- tät (z.B. das Vorliegen von Autoantikörpern oder klinischen Merkmalen), ohne die diagnostischen Kriterien für eine bestimmte Erkrankung des rheumatischen Formenkreises zu erfüllen. Diese Fälle wurden unter dem Oberbegriff «interstitielle Pneumonie mit autoimmunen Merkmalen» (IPAF) zusammengefasst, um die Forschungsagenda für diese undifferenzierten ILD-Formen voranzutreiben und zur Klärung der diagnostischen und therapeutischen Implikationen beizutragen [4]. Weitere fibrotische Lungenerkrankungen, die häufig mit Immunsuppressiva behandelt werden, sind Hypersensitivitätspneumonitis und Sarkoidose. Die verschiedenen Erkrankungen können sich hinsichtlich des Ausmaßes des Entzündungsgeschehens unterscheiden, und auch der Grad der Immunkompetenz zur Abwehr von Infektionen kann unterschiedlich sein. Daher sind hier individualisierte Therapiekonzepte erforderlich. Zwar sind auf der Grundlage groß angelegter Studien Behandlungsleitlinien für verschiedene, mit ILD assoziierte systemische Erkrankungen information@karger.com @ 2021 S. Karger GmbH, Freiburg www.karger.com/kai

Karger ${ }^{\prime \prime}=$
Prof. Antje Prasse

Abteilung für Pneumologie

Medizinische Hochschule Hannover (MHH), OE6870

Carl-Neuberg-Str. 1, 30625 Hannover, Deutschland

prasse.antje@mh-hannover.de 
ausgearbeitet worden, doch zu den Auswirkungen dieser Therapien im Kontext bestehender ILD liegt - außer zur SSc-ILD nicht viel Evidenz vor.

2014 wurde eine neue Klassifikation von langwirksamen Antirheumatika (DMARD; disease-modifying antirheumatic drugs) eingeführt [5]: Synthetische DMARD (sDMARD) wurden unterteilt in solche, die ohne Kenntnis der spezifischen Zielstruktur entwickelt wurden und eingesetzt werden (konventionelle sDMARD (csDMARDs); z.B. Azathioprin oder Methotrexat (MTX)), und solche, die von vornherein gegen eine konkrete Zielstruktur gerichtet waren (z.B. Tofacitinib), die zielgerichteten (targeted) sDMARD (tsDMARD). Biologische DMARD (bDMARD) wiederum sind gegen bestimmte, genau definierte Moleküle gerichtete Wirkstoffe, bei denen man unterscheidet zwischen neu entwickelten Substanzen (z.B. Infliximab), den sogenannten biologischen Originator-DMARD (boDMARD), und solchen, die im Prinzip die Struktur einer solchen Ursprungsoder Originator-Substanz nachahmen, den sogenannten Biosimilar-DMARD (bsDMARD).

In der vorliegenden Übersichtsarbeit fassen wir die Evidenz zu synthetischen oder biologischen DMARD zusammen, die derzeit zur Anwendung bei CTD-ILD, Sarkoidose, IPAF und fibrosierender chronischer Hypersensitivitätspneumonitis verfügbar sind (Suppl. Tab. 1) oder erforscht werden (Suppl. Tab. 2).

\section{Konventionelle synthetische DMARD}

\section{Azathioprin}

Azathioprin ist ein nichtselektiver Wirkstoff, der die Proliferation von B- und T-Zellen bremst, indem er die Purinsynthese und DNA-Replikation der Lymphozyten blockiert [6]. Azathioprin wird im Allgemeinen gut vertragen; Nebenwirkungen treten dosisabhängig und meist transient in Form von Übelkeit, Diarrhö oder erhöhten Leberenzymwerten auf. In Kombination mit Harnsäuresenkern wie Allopurinol oder Febuxostat können erhöhte Konzentrationen des aktiven Metaboliten 6-Mercaptopurin jedoch eine schwere Knochenmarksuppression verursachen. Auch eine geringe Aktivität der Thiopurin-S-Methyl-Transferase (TPMT) kann ähnliche Nebenwirkungen hervorrufen, und einige Zentren führen vor Einleitung einer Azathioprin-Behandlung regelmäßig Tests durch $[7,8]$. In Studien wurden typischerweise Dosen im Bereich von 2-2,5 mg/kg Körpergewicht (KG) angewendet, mithin wurden üblicherweise $150-200 \mathrm{mg} / \mathrm{Tag}$ verabreicht. Die PANTHER-Studie aus dem Jahr 2012 ergab für den Wirkstoff eine erhöhte Sterblichkeit bei IPF [2], während er bei ILD im Zusammenhang mit Autoimmunerkrankungen einer der am häufigsten eingesetzten steroidsparenden DMARD ist. Obwohl die Anwendung bei ILD so weit verbreitet ist, gibt es nur wenige Daten dazu. Prospektive Studien wurden lediglich zur Behandlung der SSc durchgeführt. In einer multizentrischen randomisierten kontrollierten Studie (RCT) (FAST) erhielten 45 Patienten für 6 Monate eine Induktionstherapie mit Cyclophosphamid (CYC), gefolgt von entweder Azathioprin (2,5 mg/kg KG) oder Placebo [9]. Die Studie zeigte einen Trend zur besseren Auf- rechterhaltung von Verbesserungen der forcierten Vitalkapazität (FVC) nach CYC, wenngleich dieser nicht statistisch signifikant war $(p=0,08)$, bei insgesamt guter Verträglichkeit von Azathioprin. Eine weitere prospektive (allerdings nicht verblindete) Studie zum Vergleich von CYC oral mit Azathioprin bei früher diffuser SSc bei 60 Patienten ergab hingegen eine signifikante Abnahme der FVC unter Azathioprin [10]. Seitdem wird Azathioprin gewöhnlich als Erhaltungstherapie nach einer Induktionstherapie mit stärkeren Immunsuppressiva erprobt. Unter einem solchen Protokoll zeigte eine multizentrische Analyse eine Verbesserung oder Stabilisierung der FVC bei 52\% der Patienten [11]. Neben der Evaluierung bei SSc erfolgten retrospektive Vergleiche von Azathioprin mit Mycophenolatmofetil (MMF) in einer gemischten CTD-ILD-Kohorte mit 97 Patienten [12] sowie bei mit IIM assoziierter ILD [13]; sie ergaben eine vergleichbare Wirksamkeit im Hinblick auf die Stabilisierung der FVC, jedoch eine insgesamt höhere Rate an unerwünschten Ereignissen unter Azathioprin. Bei einer anderen Kohorte mit IIM-assoziierter ILD zeigten sich ähnliche Ergebnisse im Vergleich zu CYC und MMF, jedoch mit der geringsten Toxizität unter Azathioprin [14]. Bei steroidabhängiger Sarkoidose wurde Azathioprin mit MTX verglichen; die große, multizentrische, retrospektive Studie mit 200 Teilnehmern (55 unter Azathioprin) ergab vergleichbare steroidsparende Effekte und Verbesserungen der FVC und FEV (Ein- $^{-}$ sekundenkapazität) [15]. Den vorliegenden prospektiven Daten zur SSc nach zu urteilen wird Azathioprin als steroidsparendes Mittel bei SSc in Betracht gezogen, wenn MMF nicht vertragen wird. Laut der Evidenz aus verschiedenen retrospektiven Studien kann Azathioprin als steroidsparendes Mittel bei Sarkoidose, Hypersensitivitätspneumonitis und fibrotischer CTD-ILD einschließlich systemischem Lupus erythematodes (SLE), Mischkollagenose (MCTD), Sjögren-Syndrom (SjS), IIM und RA eingesetzt werden.

\section{Methotrexat}

MTX ist ein Antifolat-Metabolit, der seine entzündungshemmenden Wirkungen über eine Kombination aus Suppression von Transmethylierungsreaktionen, Inhibition der Purin- und Pyrimidinsynthese, Förderung der Adenosinfreisetzung mit Adenosin-vermittelter Suppression des Entzündungsgeschehens und Reduktion der antigenabhängigen T-Zell-Proliferation ausübt [16]. MTX ist mit akut auftretender ILD in Verbindung gebracht worden, und seine historische Assoziation mit der ILD-Entwicklung bei RA hat zweifellos dafür gesorgt, dass es in prospektive Untersuchungen zu verschiedenen Formen fibrotischer ILD nicht eingeschlossen wurde. Studien deuten darauf hin, dass das Risiko für eine MTX-Pneumonitis bei älteren Menschen, Rauchern und Menschen mit vorbestehenden fibrotischen Lungenläsionen am höchsten ist $[17,18]$. Aktuelle Evidenz deutet auf eine fallende Rate MTX-assoziierter Pneumonitis hin, sogar eine Verzögerung der Entwicklung von RA-ILD ist möglich [19, 20]. MTX gilt als erste Wahl für eine steroidsparende Zweitlinientherapie bei steroidabhängiger Sarkoidose; die Grundlage hierfür ist eine RCT, die ergab, dass eine schnellere Steroidreduktion unter MTX im 
Vergleich zu Placebo bei guter Verträglichkeit möglich ist [21]. In der vorgenannten Studie wurde es bei Sarkoidose retrospektiv mit Azathioprin verglichen [15]. Die MTX-Behandlung beginnt in der Regel mit einer Dosis von $10 \mathrm{mg}$ oral oder subkutan 1-mal wöchentlich, die Höchstdosis liegt bei 15-20 mg. Insgesamt ist MTX das Mittel der Wahl für die Zweitlinientherapie bei steroidrefraktärer oder -abhängiger Sarkoidose [22].

\section{Leflunomid}

Leflunomid ist ein gegen Lymphozyten gerichteter Arzneistoff, der durch Hemmung der Dihydroorotat-Dehydrogenase die Denovo-Synthese von Desoxyuridin-Monophosphat (dUMP) inhibiert. Hierbei ist zu unterstreichen, dass Leflunomid nicht als tsDMARD gilt, weil seine genaue Zielstruktur erst nach der Entwicklung und dem Beginn der klinischen Anwendung bekannt wurde [5]. Leflunomid supprimiert selektiv lymphozytäre Reaktionen von aktiven (nicht aber von ruhenden) T-Lymphozyten, bei denen in Abwesenheit von intrazellulärem dUMP die p53vermittelte Apoptose ausgelöst wird [23]. Ähnlich wie MTX wurde die Anwendung von Leflunomid bei RA lange Zeit mit pulmonalen Komplikationen in Verbindung gebracht, doch auch hier deuten aktuelle Daten darauf hin, dass Leflunomid bei ILD kein zusätzliches Risiko birgt, wenngleich seltene Fälle von akuter Pneumonitis auftreten [24]. Bei ILD wird Leflunomid meist als steroidsparendes Mittel eingesetzt [25, 26], zeigte aber in einer retrospektiven Analyse auch ähnliche Wirksamkeit wie MTX bei RA-ILD [27]. Leflunomid wird in einer Dosierung von $20 \mathrm{mg}$ oral 1-mal täglich angewendet, wobei einige Zentren eine Aufsättigungsdosis von $100 \mathrm{mg}$ in den ersten 3 Tagen anwenden [25]. Die sehr begrenzten verfügbaren Daten deuten darauf hin, dass Leflunomid als steroidsparende Zweitlinientherapie bei Sarkoidose eingesetzt werden kann, wenn MTX und Azathioprin nicht vertragen werden [22], sowie bei RA-ILD und SjS, für die Leflunomid aktuell empfohlen wird, obwohl es an dedizierter Evidenz zur Wirksamkeit bei durch ILD komplizierter SjS mangelt [28].

\section{Mycophenolatmofetil}

MMF inhibiert die De-novo-Synthese von Guanin-Nukleotiden durch Hemmung der Inosinmonophosphat-Dehydrogenase, auf die Lymphozyten im Gegensatz zu vielen anderen Zelltypen ausschließlich angewiesen sind, um Guanin-Nukleotide zu synthetisieren [29]. Bemerkenswerterweise hemmt MMF laut In-vitround klinischem Nachweis nicht nur die Proliferation von Lymphozyten, sondern auch die von Fibroblasten in dosisabhängiger Weise [29]. MMF hat zahlreiche Nebenwirkungen, am häufigsten gastrointestinale Ereignisse wie Diarrhö, Übelkeit und Erbrechen [29]. Die Wirksamkeit von MMF bei ILD ist, ähnlich wie die von Azathioprin und CYC, am besten bei SSc-ILD untersucht. In der Scleroderma Lung Study (SLS) II erhielten 142 Patienten mit SScILD nach Randomisierung entweder MMF mit einer Zieldosis von 1500 mg 2-mal täglich oder CYC oral (2 mg/kg KG pro Tag); die MMF-Gruppe zeigte eine vergleichbare Verbesserung der FVC bei weniger Todesfällen und unerwünschten Ereignissen [30]. Der Vergleich der aggregierten Placebo-Patienten aus SLS I mit den Patienten aus SLS II ergab ebenfalls Verbesserungen der FVC und DLCO (Diffusionskapazität der Lunge für Kohlenmonoxid) unter MMF, nicht jedoch unter Placebo [31]. Die aktuelle multizentrische RCT SENSCIS, in der Nintedanib als antifibrotisches Mittel bei SSc-ILD untersucht wurde, ergab sowohl in der Nintedanib-als auch in der Placebo-Gruppe einen signifikant geringeren FVC-Rückgang, wenn die Patienten zusätzlich MMF erhielten [32]. Um die wahre Auswirkung auf die Progression der SSc-ILD zu evaluieren, wird in der Studie SLS III, für die die Rekrutierung derzeit läuft, die Gabe von Pirfenidon oder Placebo zusätzlich zur MMF-Therapie untersucht (NCT03221257). AuBerhalb der SSc-ILD zeigte MMF auch eine stabilisierende Wirkung bei gemischten CTD-ILD-Kohorten $[12,33]$ und eine Verbesserung der Lungenfunktionsprüfung bei IIM-ILD [13] sowie bei chronischer Hypersensitivitätspneumonitis [34, 35]. Bei Patienten mit IPAF zeigte sich unter MMF im Vergleich zu Kontrollen, die kein MMF erhielten, ein Trend zu einer langsameren Verschlechterung der DLCO und FVC, der Effekt war jedoch statistisch nicht signifikant [36]. Aufbauend auf die hochwertige Evidenz aus den SENSCIS- und SLS-II-Studien und das insgesamt gute Sicherheitsprofil ist MMF heute bei fibrotischer CTDILD sehr gebräuchlich, mit Ausnahme von RA-ILD aufgrund mangelnder Wirksamkeit bei Arthritis [37], und auch bei fibrotischer Sarkoidose und chronischer Hypersensitivitätspneumonitis kann es in Betracht gezogen werden [38].

\section{Calcineurin-Inhibitoren (FK506 und Cyclosporin A)}

Sowohl Tacrolimus (FK506) als auch Cyclosporin A (CSA) wirken inhibierend auf T-Zellen, indem sie Calcineurin hemmen und so die Interleukin (IL)-2-abhängige T-Zell-Differenzierung herunterregulieren [29]. Ihre Anwendung ist am besten beschrieben und weit verbreitet in der Prävention der Transplantatabstoßung nach der Allotransplantation von soliden Organen, insbesondere von Herz und Lunge. Nebenwirkungen treten bei praktisch allen Patienten auf, unter anderem Nierentoxizität, Anämie, Leukozytopenie und Infektionen, was eine aufmerksame und strenge Dosisanpassung anhand der Messung der Talspiegel erfordert. Bei ILD sind beide Wirkstoffe am gründlichsten bei Patienten mit entzündlicher Myopathie-assoziierter ILD und Anti-SynthetaseSyndrom untersucht worden, insbesondere bei schnell fortschreitender ILD als Salvage-Therapie mit hoher kurzfristiger Mortalität [39]. Die zusätzliche Gabe von Calcineurin-Inhibitoren (CNI) hatte einen positiven Effekt auf die Lungenfunktion [40-44] und das Überleben [39, 45]; alle Studien hierzu waren retrospektiv und sehr heterogen in Bezug auf die Geschwindigkeit der ILD-Progression, die Erfassung und Art der Anti-Synthetase-Antikörper sowie die gleichzeitige Anwendung anderer DMARD. In einer prospektiven Pilotstudie mit 10 Patienten, die eine akute oder subakute ILD in Zusammenhang mit einer entzündlichen Myopathie hatten, wurde CSA zusammen mit hochdosiertem Prednisolon und intravenösem CYC angewendet; die 3-Monats-Mortalität betrug hierbei 50\% [46]. Dieses Vorgehen wird bei schnell fortschreitender ILD und entzündlicher Myopathie, die gegen CYC oder Rituximab (RTX) refraktär sind, in einer aktuellen Pu- 
blikation der European Respiratory Society (ERS) empfohlen [47]. Die Zielwerte für die FK506-Talspiegel lagen in den Studien im Bereich von 5-20 $\mu \mathrm{g} / \mathrm{l}$, sollten aber bei Ansprechen auf 5-8 $\mu \mathrm{g} / \mathrm{l}$ herabgesetzt werden. Ebenso lagen die CSA-Zielwerte in den Studien bei 100-200 $\mu \mathrm{g} / \mathrm{l}$. Der Evidenz zufolge ist der Einsatz von CNI auf schnell fortschreitende IIM-assoziierte ILD beschränkt, in der Regel als Zusatztherapie zu einem anderen DMARD wie MMF. In diesem Kontext ist Tacrolimus aufgrund seines insgesamt besseren Sicherheitsprofils zu bevorzugen, sollte aber bei fehlendem Nachweis einer Stabilisierung der Erkrankung abgesetzt werden. Einige Experten betrachten es primär als Notfalltherapie bei refraktärer Erkrankung nach Vorbehandlung mit CYC oder RTX [47].

\section{Cyclophosphamid}

Neben Steroiden war CYC der erste zytotoxische Wirkstoff, der zur Behandlung von progressiver ILD eingesetzt wurde. Es ist ein alkylierendes Mittel, das die lymphozytäre Aktivität sowohl im peripheren als auch im zentralen lymphatischen Gewebe herabsetzt und durch Reduktion der Bildung von Monozyten-Vorläuferzellen den Einstrom und die Aktivierung von Makrophagen unterdrückt. Sowohl die humorale als auch die zellvermittelte Immunantwort werden durch CYC inhibiert [48]. Von allen hier behandelten konventionellen Wirkstoffen ist CYC einer der stärksten, aber mit Sicherheit auch der mit dem ungünstigsten Sicherheitsprofil, da er häufig Leukozytopenie und Infektionen, weniger häufig auch Sekundärtumoren und maligne hämatologische Erkrankungen sowie eine Gonadeninsuffizienz verursacht [48]. In früheren Studien war die orale Einnahme der bevorzugte Verabreichungsweg, doch da dies mit einer höheren kumulativen Dosis und Nebenwirkungsrate assoziiert ist, wird CYC heute in der ILD-Behandlung in Europa nur noch selten oral und stattdessen als intravenöse Pulstherapie eingesetzt. Erstmals wurde in der multizentrischen RCT SLS I eine moderate Verbesserung der Lungenfunktion bei SSc-ILD für CYC ( $2 \mathrm{mg} / \mathrm{kg} \mathrm{KG}$ oral) im Vergleich zu Placebo über einen Studienzeitraum von 6 Monaten nachgewiesen [49]. 6 Monate nach dem CYC-Behandlungsende hatte der positive Effekt auf die FVC jedoch keinen Bestand mehr [49]. Im selben Jahr wurde die FAST-Studie publiziert, in der Prednisolon mit intravenösem CYC $\left(0,6 \mathrm{~g} / \mathrm{m}^{2}\right.$ alle 4 Wochen für 6 Zyklen) gefolgt von Azathioprin oder Placebo verglich wurde; sie ergab eine statistisch nicht signifikante Verbesserung der FVC um 4,2\% in der Behandlungsgruppe [9]. Diese Studien bilden noch heute die Grundlage für die geltende Empfehlung vom Evidenzgrad A der European League Against Rheumatism (EULAR) für die Anwendung von CYC bei SSc-ILD [50]. CYC wurde außerdem retrospektiv in verschiedenen Anwendungsgebieten untersucht - bei schnell fortschreitender nichtspezifischer interstitieller Pneumonie (NSIP), wo es zur Stabilisierung der Erkrankung führte [51], ebenso wie bei akut exazerbierender RA-ILD [52] und diversen anderen progressiven ILD [53], wo es besondere Wirksamkeit bei lymphozytärer interstitieller Pneumonie (LIP) und NSIP zeigte [53]. Eine aktuelle Metaanalyse ergab bei CTD-ILD insgesamt eine Verbesserung der FVC (nicht aber der DLCO) un- ter CYC im Vergleich zu Placebo, jedoch keinen klaren Nutzen im Vergleich zu MMF [54]. Bei entzündlicher Myositis-assoziierter ILD wird CYC in der Regel nur als Salvage-Therapie in Betracht gezogen und lediglich sehr kleine Kohortenstudien oder anekdotische Berichte weisen auf eine Verbesserung bei 50\% der Patienten hin [39]. Bei intravenöser Gabe wird das in der FAST-Studie angewendete Protokoll (0,6 g/m $\mathrm{m}^{2}$ Körperoberfläche alle 4 Wochen für 6 Zyklen [9]) meist zusammen mit Mesna (2-Mercaptoethansulfonat-Natrium) angewendet. Dasselbe Protokoll bei CTD-ILD wird derzeit in der RECITAL-Studie im Vergleich zu RTX untersucht [55]. Unterm Strich ist CYC einer der wenigen konventionellen Wirkstoffe, zu denen RCT-Daten als therapeutische Entscheidungsgrundlage vorliegen. Es ist für die Anwendung bei SScILD zugelassen, doch potenzielle Nebenwirkungen wie solide und hämatologische maligne Erkrankungen, schwere Infektionen und Gonadeninsuffizienz limitieren die Einsatzbarkeit bei der größtenteils weiblichen und jüngeren SSc-Population [54, 56]. CYC wird für die Salvage-Therapie bei CTD-ILD eingesetzt, doch hierbei muss gegen die potenziellen Auswirkungen im Hinblick auf eine Lungentransplantation und gegen das Nebenwirkungsrisiko abgewogen werden.

\section{Zielgerichtete synthetische DMARD}

\section{JAK/STAT-Inhibitoren}

Der nach den JAK (Januskinasen) und STAT (Signal Transducers and Activators of Transcription) benannte Signalweg ist als zentrale Konvergenzstrecke der extrazellulären, zytokinvermittelten Übermittlung von Signalen an den Zellkern auch an zahlreichen Immun- und Autoimmunerkrankungen beteiligt [57]. In den letzten Jahren sind selektive JAK-1- und JAK-2-Inhibitoren zur Behandlung unterschiedlicher Autoimmunerkrankungen zugelassen worden, von der Myelofibrose über Polycythaemia vera und Colitis ulcerosa bis hin zu Psoriasis und RA [57]. Die Untersuchung des Sicherheitsprofils in der Langzeit-Verlängerungsstudie ORAL Sequel ergab schwerwiegende unerwünschte Ereignisse in Form von Infektionen bei 9\% (davon 24\% Pneumonien) sowie Erkrankungen des Muskel-Skelett-Systems und des Bindegewebes bei 5,5\% der Patienten [58]. Bei ILD wurde dieser Behandlungsansatz als Salvage-Therapie bei schnell fortschreitender ILD im Zusammenhang mit anti-MDA5-amyopathischer Dermatomyositis (Anti-MDA5-ADM) trotz Therapie mit hochdosierten Steroiden, CSA und CYC untersucht; hier wurde eine historische Mortalität von bis zu 50\% berichtet [59]. Basierend auf einer Reihe ungünstiger Prognosefaktoren, die aus historischen Kontrollen mit einer Mortalität von 100\% hergeleitet wurden, überlebten 3 von 5 der mit Tofacitinib (10 mg/Tag) behandelten Patienten und zeigten Besserung [60]. Durch diese Daten ermutigt wurde in einer prospektiven, nicht verblindeten, monozentrischen Studie Tofacitinib bei 18 Patienten mit früher AntiMDA5-ADM-ILD untersucht; sie ergab eine 6-Monats-Überlebensrate von 100\% im Vergleich zu 78\% bei nach Therapiestandard behandelten historischen Kontrollen, begleitet von Verbesserungen der FVC und DLCO [61]. Bei RA wird diese Therapie weithin 
angewendet, obwohl bisher nur vorläufige retrospektive unkontrollierte Daten zu 15 Patienten vorliegen, die von einer signifikanten Verbesserung der DLCO und einer Stabilisierung der Fibroseausdehnung in der hochauflösenden Computertomografie (CT) nach 12 Monaten berichten [62]. In der kürzlich begonnenen randomisierten Studie PULMORA (NCT04311567) sollen die Auswirkungen auf die Entwicklung der ILD bei RA untersucht werden. Angesichts früher, aber vielversprechender Berichte über die Wirksamkeit von Tofacitinib bei kutaner Sarkoidose [63] werden außerdem in einer Phase-1-Studie das klinische Ansprechen und die STAT-abhängige Genexpression unter Tofacitinib auch bei pulmonaler Sarkoidose untersucht (NCT03793439). Während die Ergebnisse dieser Studien mit Spannung erwartet werden, kann Tofacitinib derzeit bei Anti-MDA5-ILD, die trotz CYC oder RTX weiter fortschreitet, in Betracht gezogen werden.

\section{Biologische DMARD}

\section{Rituximab}

RTX ist ein monoklonaler Antikörper gegen CD20, das auf BVorläuferzellen und reifen B-Lymphozyten exprimiert wird. Der Antikörper wird seit Jahrzehnten in unterschiedlichen Dosen und Anwendungsarten in der Behandlung vieler verschiedener Autoimmun- und hämatologischer Erkrankungen eingesetzt [64]. Er induziert die Depletion von B-Zellen über mehrere Mechanismen, einschließlich antikörperabhängiger zellvermittelter Toxizität, Apoptose und komplementvermittelter Zytotoxizität [65]. Obwohl sein direktes Ziel die B-Zellen sind, ist auch über therapeutische Effekte auf T-Zellen berichtet worden. RTX ist von der US-Zulassungsbehörde FDA (Food and Drug Administration) zur Anwendung bei RA und Granulomatose mit Polyangiitis zugelassen, es wird zulassungsüberschreitend aber auch bei praktisch jeder Autoantikörper-assoziierten ILD angewendet. Nur eine einzige RCT wurde bisher dazu abgeschlossen und publiziert; hier erhielten 16 Patienten mit früher SSc RTX in einer Dosis von je $1000 \mathrm{mg}$ an Tag 0, Tag 14 und nach 6 Monaten und zeigten einen statistisch nicht signifikanten Trend zur Verbesserung der FVC [66]. Zwei prospektive, nicht kontrollierte, nicht verblindete Studien bei diffuser SSc mit insgesamt 32 Teilnehmern ergaben einen günstigen Effekt auf die FVC, entweder eine Verbesserung oder eine Stabilisierung $[67,68]$, und eine nicht verblindete Vergleichsstudie ergab eine Verbesserung der FVC unter RTX, nicht aber unter intravenösem CYC [69]. Die neuere Studie bewertete die Wirksamkeit von RTX zusammen mit MMF und zeigte auch eine Verbesserung der Fibroseausdehnung laut CT [68]. Weitere retrospektive Studien mit meist gemischten Kohorten von SSc-Patienten mit oder ohne evidente ILD ergaben durchgängig bei der Mehrzahl der Patienten eine Stabilisierung oder Verbesserung der Lungenfunktion [70-72]. Zur RA-ILD gab es eine prospektive, nicht verblindete Studie, in der RTX zusammen mit MTX in einer Dosis von 1000 mg an Tag 0, Tag 15, in Woche 24 und in Woche 26 bei insgesamt 10 Patienten angewendet wurde, von denen 7 in Woche 48 eine Stabilisierung der DLCO, FVC und der CT-Merkmale der ILD zeigten [73]. Retrospektive Berichte belegen ebenfalls eine Stabilisierung der Lungenfunktion [74] und ganz aktuell auch bei Patienten mit progressiver RA-ILD vor der RTX-Therapie, die dann unter dieser Therapie eine Besserung zeigten [75]. Insgesamt scheint das Erscheinungsbild einer NSIP im CT-Scan und in der Histopathologie (mit Infiltraten CD20positiver Lymphozyten) die Wahrscheinlichkeit des Therapieansprechens zu erhöhen, während das Vorliegen einer gewöhnlichen interstitiellen Pneumonie (UIP) mit schlechten Ansprechraten korreliert [74, 76]. Die Anwendung von RTX wurde auch bei Patienten mit IIM mit Anti-tRNA-Synthetase-Antikörpern beschrieben, einer sich oft schnell entwickelnden und stark entzündlichen Erkrankung. In einer nicht verblindeten, prospektiven, multizentrischen Phase-2-Studie wurden 12 Patienten mit Anti-Synthetase-Syndrom-assoziierter ILD, die gegen Steroide und $\geq$ DMARDs refraktär waren, mit RTX behandelt (1000 mg an Tag 0, Tag 15 und nach 6 Monaten). Die Ergebnisse belegen insgesamt signifikante Verbesserungen der Muskelkraft, eine Kreatinkinase-Normalisierung, Steroidreduktion und Stabilisierung oder Verbesserung bei 90\% der Patienten [77]. Vergleichsweise große multizentrische retrospektive Studien zum Anti-Synthetase-Syndrom in Verbindung mit progressiver ILD belegen eine ähnliche Wirksamkeit [78, 79]. Aufgrund dieser Studienergebnisse ist RTX derzeit das Mittel der Wahl bei refraktärer und progressiver IIM-ILD (oder beim Anti-Synthetase-Syndrom). Eine separate retrospektive Studie bei Patienten mit primärer SjSassoziierter ILD ergab eine signifikante Verbesserung der FVC und DLCO bei 10 Patienten [80]. Nachdem weitere retrospektive Studien zu gemischten CTD-ILD-Kohorten insgesamt günstige Effekte auf die Lungenfunktion belegen [80-85], werden die Ergebnisse von 2 RCT, die derzeit rekrutieren und in denen RTX versus CYC intravenös (RECITAL; NCT01862926) [55] bzw. MMF plus RTX versus MMF plus Placebo (EVER-ILD; NCT02990286) bei CTD-ILD untersucht werden soll, mit Spannung erwartet. RTX ist bei diesen Patienten mit potenziell letalen Infektionen assoziiert [79], und eine Prophylaxe der Pneumocystis-Pneumonie (PCP) wird in der Regel empfohlen, insbesondere bei gleichzeitiger Anwendung weiterer Immunsuppressiva. Zusammenfassend lässt sich sagen, dass RTX die beste therapeutische Option bei schweren und sich schnell entwickelnden, entzündlichen ILD ist, die mit einer Akkumulation von B-Zellen einhergehen.

\section{Tocilizumab}

Tocilizumab ist ein humanisierter monoklonaler Antikörper, der als IL-6-Rezeptorantagonist wirkt. IL-6 ist ein pleiotropes proinflammatorisches Zytokin, das zur Pathogenese der RA beiträgt, aber auch mit der ILD-Progression bei SSc assoziiert ist [86, 87]. Tocilizumab ist weithin gebräuchlich und zur Behandlung von mittelschwerer bis schwerer RA, systemischer juveniler idiopathischer Arthritis und Riesenzellarteriitis zugelassen [88]. In 2 aktuellen multizentrischen RCT wurde Tocilizumab bei Patienten mit SSc und Ausgangswerten der mittleren FVC und DLCO von $>80 \%$ des Sollwerts untersucht und die FVC-Verschlechterung als explorativer Endpunkt betrachtet. In der Studie faSScinate
Kompass Autoimmun 2021;3:2-8 DOI: $10.1159 / 000514258$ 
wurden 87 Patienten randomisiert und zeigten eine abgemilderte Verschlechterung der FVC mit einer Differenz von $136 \mathrm{ml}$ in Woche $24(p=0,037)$ [89]. Ähnliche Auswirkungen waren in der offenen Phase der Studie bei Patienten zu verzeichnen, die nach Woche 48 von Placebo zu Tocilizumab wechselten [90]. Vorläufige Ergebnisse der Phase-3-Studie FocuSSed mit 212 Teilnehmern belegen eine signifikante Reduktion der FVC-Verschlechterung ( $-0,6 \%$ vs. $-3,9 \%)$ zugunsten von Tocilizumab versus Placebo [91]. Beide Studien hatten Patienten mit frühem Krankheitsbeginn aufgenommen und ergaben einen signifikanten Effekt der Tocilizumab-Behandlung (erhaltene FVC) auch bei SSc-Patienten, die zu Studienbeginn als nicht an ILD erkrankt eingestuft wurden. In beiden Studien wurde Tocilizumab subkutan in einer Dosis von $162 \mathrm{mg}$ wöchentlich angewendet, schwerwiegende Infektionen traten bei $16 \%$ unter der Studienbehandlung versus $5 \%$ unter Placebo auf. Hierbei ist anzumerken, dass die CCL18-Werte, die mit der Entzündungsaktivität und dem Überleben bei SSc-ILD korrelieren [92, 93], bei den mit Tocilizumab behandelten Patienten signifikant verringert waren [89]. Diese Daten sind zwar vielversprechend im Hinblick auf die Prävention einer progressiven ILD bei SSc, jedoch liegen nur wenige bis gar keine Daten zur Wirkung von Tocilizumab auf andere klinisch signifikante und progressive ILD vor. Eine multizentrische retrospektive Studie bei 28 RA-ILD-Patienten, die mindestens 1 Dosis Tocilizumab erhielten, ergab bei der Mehrheit der Patienten eine Stabilisierung oder Verbesserung der FVC und DLCO (beide 76\%). Interessant ist hierbei, dass die meisten Patienten bei der Aufnahme in die Studie in einem frühen Krankheitsstadium waren, mit einer medianen Baseline-FVC von 99\% [94]. Abschließend lässt sich sagen, dass Tocilizumab eine vielversprechende Behandlungsoption für SSc- und potenziell auch für RA-ILD-Patienten darstellt und in frühen Krankheitsphasen mit höherer Entzündungsaktivität besonders wirksam sein könnte. Der verbreitete Einsatz von Tocilizumab bei RA erfordert prospektive Studien, um den wahren Einfluss auf den Verlauf der ILD sowie das optimale Timing zu untersuchen.

\section{TNF-a-Hemmer}

Der Tumornekrosefaktor alpha (TNF- $\alpha$ ) ist ein Schlüsselzytokin im Th1 (T-Helfer-1)-Signalweg, der letztlich in einer granulomatösen Entzündung mündet. Der bei ILD am häufigsten angewendete Inhibitor von TNF- $\alpha$ ist Infliximab, das intravenös in einer Dosierung von 3 oder $5 \mathrm{mg} / \mathrm{kg}$ KG in Woche 0, 2 und 6 und danach alle 4-8 Wochen verabreicht wird. Das Hauptanwendungsgebiet ist als Off-Label-Drittlinientherapie bei steroidabhängiger pulmonaler Sarkoidose; eine randomisierte, doppelblinde, placebokontrollierte Studie ergab hier eine Verbesserung der FVC in der Infliximab-Gruppe [95]. Infliximab tritt problemlos in das Zentralnervensystem (ZNS) über und ist dadurch bei Neurosarkoidose hochwirksam [96]. Bei schwerer Herzinsuffizienz ist Infliximab kontraindiziert, und bei bis zu 36\% der Patienten in einer multizentrischen retrospektiven Analyse traten Infektionen auf [97]. Hierbei ist jedoch anzumerken, dass die infektiösen Komplikationen sich von denen bei anderen immunsuppressiven
Therapien unterscheiden und meist von geringer Schwere sind [98]. Insbesondere Virusinfektionen wie Herpes zoster und Warzen treten bei mit Infliximab behandelten Patienten häufiger auf [99]. Es besteht ein erhöhtes Risiko, dass eine latente Tuberkulose aktiviert wird, doch dies wurde bei Sarkoidose sehr selten beobachtet $[98,100]$. Adalimumab, ein vollständig humaner AntiTNF-Antikörper, wurde ebenfalls erfolgreich in einer nicht verblindeten Studie untersucht und zeigte bei $82 \%$ der Patienten Wirksamkeit in Form von Verbesserungen bei der FVC, dem 6-Minuten-Gehtest und der Senkung der Steroiddosis [101]. Adalimumab wird subkutan verabreicht, die übliche Dosis beträgt 40 mg pro Woche. Die Rolle der TNF- $\alpha$-Blockade bei anderen Autoimmun-ILD ist noch ungeklärt, doch eine aktuelle Übersichtsarbeit zur TNF- $\alpha$-Blockade bei RA-ILD ergab eine hohe Rate von Nebenwirkungsmeldungen (87,5\%) und eine berichtete Mortalität von 35\% bei geringer Evidenz von potenziellem Nutzen in dieser Patientenkohorte, da es keine RCT gibt [102].

\section{Abatacept}

Abatacept ist ein selektiver Modulator der Kostimulation; das rekombinante Fusionsprotein enthält die extrazelluläre Domäne des humanen CTLA-4 (cytotoxic T lymphocyte-associated protein 4) und hemmt so die T-Zell-Aktivierung durch spezifische Bindung an CD80 und CD86 auf antigenpräsentierenden Zellen [103]. Es ist für die Behandlung der aktiven RA zugelassen. Eine erste Studie zur Auswertung retrospektiver, multizentrischer Registerdaten ergab eine Stabilisierung oder Verbesserung der FVC und DLCO bei mehr als $80 \%$ der 63 ausgewerteten Patienten mit RA-ILD, die mit $10 \mathrm{mg} / \mathrm{kg} \mathrm{KG}$ an Tag 0 und Tag 15 und danach alle 4 Wochen behandelt wurden [104]. Bei 17\% der Patienten wurde Abatacept aufgrund unerwünschter Ereignisse abgebrochen, darunter $3 \mathrm{~Pa}-$ tienten mit schwerwiegenden Infektionen. Eine aktuelle retrospektive Kohortenstudie mit 44 Patienten mit RA-ILD ergab vergleichbare positive Effekten auf die Lungenfunktion ohne schwerwiegende unerwünschte Ereignisse [105]. Derzeit läuft die Rekrutierung für 2 prospektive Studien zu Abatacept: In der APRIL-Studie (NCT03084419) soll in einem nicht verblindeten, 1-armigen Setting bei Patienten mit RA-ILD der Verlauf der Lungenfunktion untersucht werden, um als Machbarkeitsstudie die Grundlage für eine größer angelegte Studie zu bilden. Die Phase2-Studie ATtackMy-ILD (NCT03215927) ist eine placebokontrollierte, randomisierte Studie zur Anwendung bei Myositis-assoziierter ILD in einer Dosis von 125 mg subkutan wöchentlich. Während die Ergebnisse dieser Studien noch ausstehen, stellt Abatacept ein vielversprechendes Mittel zur Behandlung der IIM-ILD und RA-ILD dar, doch seine Wirkung auf klinische und radiologische Subtypen insbesondere bei RA-ILD bleibt noch zu klären.

\section{Schlussfolgerung}

Bei vielen systemischen Erkrankungen, insbesondere Bindegewebserkrankungen, ist eine fortschreitende ILD weiterhin der wichtigste Faktor für die Mortalität [106, 107]. Trotz dieser negativen prognostischen Bedeutung besteht der Großteil der Evi- 
denz, auf die sich die therapeutische Entscheidungsfindung stützt, aus retrospektiven oder kleinen prospektiven Studien [56] Studien, die derzeit in der Rekrutierungsphase sind (Suppl. Tab. 2), werden Aufschluss über neue Behandlungsoptionen geben und hoffentlich zur behördlichen Zulassung neuer Wirkstoffe und zu besseren langfristigen Behandlungsergebissen führen. Zahlreiche Immunmodulatoren, die bisher nicht für CTD zugelassen sind, werden derzeit bei IPF untersucht und an anderer Stelle evaluiert [108]; sie sind nicht Gegenstand des vorliegenden Artikels. Angesichts neu gewonnener Evidenz zur Wirksamkeit von antifibrotischen Wirkstoffen bei mit Autoimmunerkrankungen assoziierter ILD [109] besteht dringender Anlass zu weiterführenden Untersuchungen, um fundierte Entscheidungen über die Anwendung antiinflammatorischer und antifibrotischer Arzneimittel oder einer Kombination zu ermöglichen.

\section{Disclosure Statement}

Die Autoren haben keine Interessenkonflikte offenzulegen.

\section{Finanzierung}

Für dieses Projekt gab es keine spezifische Finanzierung. Dr. Seeliger erhält Unterstützung durch PRACTIS - Clinician-Scientist-Programm der Medizinischen Hochschule Hannover, finanziert von der Deutschen Forschungsgesellschaft (DFG, ME 3696/3-1).

\section{Beiträge der einzelnen Autoren}

B.S. führte die Literaturanalyse durch und erstellte die Erstfassung des Manuskripts. A.P. führte eine kritische Durchsicht des Manuskripts durch. Beide Autoren genehmigten die finale Fassung des Manuskripts.

\section{Supplementary Material}

Die Tabellen 1 und 2 sind als Online Supplementary Material unter $w w w$. karger.com/doi/10.1159/000514258 verfügbar.

\section{Literatur}

Die Literatur ist unter www.karger.com/doi/10.1159/000514258 abrufbar. 